\title{
A new method to select demulsifiers and optimize dosage at wet crude oil separation facilities
}

\author{
Guillaume Raynel*, Debora Salomon Marques, Sajjad Al-Khabaz, Mohammad Al-Thabet, and Lanre Oshinowo
}

Saudi Aramco, Research and Development Center, 31311, Dhahran, Saudi Arabia

Received: 25 October 2020 / Accepted: 9 December 2020

\begin{abstract}
The current practice for crude oil demulsifier selection consists of pre-screening of the best performing demulsifiers followed by field trials to determine the optimum demulsifier dosage. The method of choice for demulsifier ranking is the bottle test. As there is no standard bottle test method, there are different methodologies reported in the literature. In this work, a new approach to bottle test and field trial was described which improved significantly the selection and dosage of the demulsifier. The bottle test was optimized by measuring an accurate mass of demulsifier. This method produces repeatable results. This bottle-test methodology was benchmarked against field trial results performed in oil processing plants. The field trials were also improved to avoid the accumulation effect of demulsifier, when optimizing their dosage. The field data for the optimization of demulsifier dosage was analyzed mathematically; and a graphical method to determine the optimum range is described.
\end{abstract}

\section{Introduction}

Crude oil is typically extracted with dispersed water in varying concentrations. The Water-in-Oil (W/O) emulsions are believed to be initially formed during the migration of oil and water through the porous reservoir rocks to the production wells [1] and subsequent transport to production facilities for phase separation. Pressure drop across choke valves at production headers used to manage production flows creates significant agitation and turbulent energy further emulsifying the water and oil [2, 3]. The dispersed water droplets are stabilized by surface active compounds naturally present in the oil such as resins, asphaltenes, solid particles, organic acids and bases [4, 5]. These surfactants migrate to the oil--water interface forming a film around the water droplets slowing the natural process of coalescence between droplets [4-8]. The film significantly influences whether the emulsion separates easily (loose emulsion) or will not separate spontaneously (tight emulsion) $[8,9]$. Tight emulsions require great investment by the oil companies in processes and chemical intervention to separate, or break, the emulsion [2] to produce crude oil within export specifications, required by the recipient (i.e. other producers, transporters or refiners). These requirements are usually of less than $0.5 \mathrm{v} / \mathrm{v} \%$ in Basic Sediment and Water (BS\&W); and less than $10 \mathrm{lbs}$. of total dissolved salts Per Thousand Barrels (PTB) of oil [10].

\footnotetext{
* Corresponding author: guillaume.raynel@aramco.com
}

The stabilization of crude oil emulsions is believed to be dependent on the asphaltene solubility in the oil, the asphaltene-to-resin ratio, the $\mathrm{pH}$ and the degree of polarity of the asphaltenes and resins [11]. The composition of crude oils can vary greatly from one producing field to another, resulting in different combinations of these parameters with unique effects. In some particularly tight oil emulsions, water cannot be separated solely by physical methods, such as, retention, heating and electrocoalescence [12]. In such cases, a combination of physical and chemical methods is required to achieve the desired water separation. Zolfaghari et al. [4] published a comprehensive review of different demulsification methods and mechanisms of demulsifier action.

For decades, demulsifiers have been designed to break crude oil-water emulsions. The proper demulsifier selection is essential to crude oil production. The demulsifier dosage affects directly the crude oil quality exiting the production facility to the refinery or export [13]. No single demulsifier can break all crude oil emulsions with the same water separation performance due to the vast crude oil and water compositional differences. Demulsifiers are formulated from various polymers, such as alkoxylated phenolic resins, to specifically address two factors: water separation efficiency, and the separation rate. The separation efficiency is the amount of water removed from the emulsion. The separation rate is the speed at which the emulsion resolves in two phases, oil and water. For a specific production facility, the demulsifier can be selected based on the residence time 
in each separation vessel and desired water removal, or water dropout, from the different separation vessels $[12,14]$.

The bottle test is the traditional method used to assess oil emulsion stability $[2,12,13,15]$. The bottle test is a simple assessment of the rate of emulsion separation by visual observation of the separated volume of water with time. The bottle test does not take into consideration the "live" production stream pressure or the geometric and flow conditions in the separation vessels. Many authors have published experimental protocols used to perform bottle tests $[12,14]$. The bottle test is used to select an effective demulsifier. Following the bottle test demulsifier screening to select a short-list of trial candidates, a final demulsifier selection is made following field trials of the short-listed demulsifiers at a large scale in the actual production facilities. Bottle tests have an important role selecting the best demulsifier formulations to reduce the time and cost of field trials.

In this paper, the demulsifier selection for a Saudi Arabian oil production facility based on bottle testing and field trials is described. The limitations of the bottle test are described and a set of best practices to improve the reliability of the results are recommended. The data generated during field trials by the production facility is analyzed to identify an important effect of the sequence of demulsifier dosages. Finally, a mathematical formula of the demulsifier performance is used to determine the optimum demulsifier dosage.

\section{Materials and methods}

A precision balance (PRECISA XT220A-FR) with a closed glass-cover was used to weigh the demulsifier with an accuracy of one tenth of milligram. Figure 1 shows a picture of 100-mL glass centrifuge tubes from KIMBLE (KIMAX 28-410) used in the bottle test with dissimilar graduated scales, as described in Table 1. The tubes have a screwcap with a PTFE liner to reduce the loss of light hydrocarbon components during the experiment. The crude oil specific gravity was measured at $15.56{ }^{\circ} \mathrm{C}\left(60^{\circ} \mathrm{F}\right)$ using a density meter with an oscillating U-tube (DMA 5000M ANTON PAAR), as described in ASTM D4052-18a [16]. The API gravity is calculated from the measured specific gravity. The Arabian medium crude oil used in this study has an API gravity of $28.8^{\circ}$. A thermostatic oven (Lindberg/Blue M Laboratory Gravity Oven Model GO1330A) was used to maintain a sample temperature of $30{ }^{\circ} \mathrm{C}$ during the bottle test.

A Saturate, Aromatic, Resin and Asphaltene (SARA) analysis was performed. The quantity of asphaltene was determined by precipitation with 30 volumes of cold n-pentane $\left(3{ }^{\circ} \mathrm{C}\right)$. The composition of the de-asphaltenated filtrate was obtained by open column chromatography on a silica gel column sequentially eluted with n-pentane, a 50:50 mixture of n-pentane and dichloromethane, and a 15:15:70 mixture of methanol, acetone and chloroform to isolate saturated hydrocarbons, aromatics and non-asphaltenic polar compounds - Nitrogen, Sulfur and Oxygen (NSO) compounds. Dichloromethane (Sigma Aldrich), n-pentane

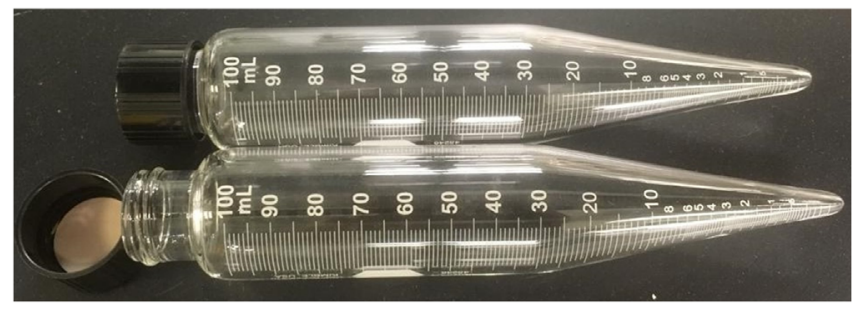

Fig. 1. Two 100-mL centrifuge tubes used for bottle testing.

Table 1. 100-mL KIMBLE centrifuge tube graduation scales.

\begin{tabular}{lc}
\hline Graduations $(\mathrm{mL})$ & Scale $(\mathrm{mL})$ \\
\hline $0-1$ & 0.05 \\
$1-3$ & 0.1 \\
$3-6$ & 0.2 \\
$6-10$ & 0.5 \\
$10-100$ & 1 \\
\hline
\end{tabular}

(Sigma Aldrich), methanol (Sigma Aldrich), chloroform (Sigma Aldrich), acetone (Sigma Aldrich), and silica gel (Sigma Aldrich) were used without further purification. The Arab medium crude oil is composed of $30 \mathrm{wt} \%$ Saturated hydrocarbons, $34 \mathrm{wt} \%$ Aromatics, $11 \mathrm{wt} \%$ Resins (NSO), and $25 \mathrm{wt} \%$ Asphaltenes. The wet crude oil emulsion is highly stable up to a high temperature of $80^{\circ} \mathrm{C}$. The water droplets preferably sediment, as opposed to coalesce. This sedimentation forms a sludge at the bottom of the container, which was difficult to re-homogenize after a week. The water cut of this emulsion was $24 \mathrm{vol} \%$.

\section{Experimental}

\subsection{Bottle test improvements}

The bottle test technique is used for selecting demulsifiers at the production facility by measuring the water separation performance on crude oil emulsions [12]. Extensive studies in the past have shown that this experimental method is affected by various physical and chemical parameters: salinity [17-19], $\mathrm{pH}[20,21]$, wall and boycott effect [22], temperature [17, 21], ageing [23], shaking/mixing $[2,24]$ and diluent $[24,25]$. The procedure used in this work has similarities and differences to procedures reported in the literature and is listed in bold text in Table 2 .

The following improvements in the methodology of the bottle test experiment were established in this study:

- Chemical integrity of the sample

All bottle tests were performed in glass centrifuge tubes with a screwcap to avoid the loss of light components, like pentanes and hexanes, from the wet crude oil emulsion. These volatile compounds affect the bulk viscosity 
Table 2. Differences between bottle test techniques.

\begin{tabular}{|c|c|c|}
\hline Parameter & & Reference \\
\hline Bottle test tube & $\begin{array}{l}\text { - Tube material } \\
\text { ○ Glass } \\
\text { ○ Plastic } \\
\text { - Jar (bottle) }\end{array}$ & $\begin{array}{c}{[12,17-19,21,23] \text { and this work }} \\
{[25]} \\
{[24]}\end{array}$ \\
\hline Demulsifier addition & $\begin{array}{l}\text { - Dilution } \\
\text { o Distilled water } \\
\text { o Organic solvents } \\
\text { - Volume } \\
\text { - Mass }\end{array}$ & $\begin{array}{c}{[17-19,25]} \\
{[12,21]} \\
{[24]} \\
\text { This work }\end{array}$ \\
\hline Mixing method & $\begin{array}{l}\text { - Table shaker } \\
\text { - Homogenizer } \\
\text { - Hand shaker }\end{array}$ & $\begin{array}{c}{[12,17,21,24]} \\
{[18,20,21,23,25]} \\
{[19] \text { and this work }}\end{array}$ \\
\hline Emulsion & $\begin{array}{l}\text { - Fresh (from process) } \\
\text { - Synthetic (made at the lab) }\end{array}$ & $\begin{array}{c}{[12,24] \text { and this work }} \\
{[17,19-21,23,25]}\end{array}$ \\
\hline Temperature control & $\begin{array}{l}\text { - Thermostatic bath } \\
\text { - Thermostatic oven } \\
\text { - Room temperature }\end{array}$ & $\begin{array}{c}{[12,17,23,24]} \\
{[21] \text { and this work }} \\
{[19,20,25]}\end{array}$ \\
\hline Data processing & $\begin{array}{l}\text { - Error } \\
\text { - Correction }\end{array}$ & $\begin{array}{l}{[21] \text { and this work }} \\
\text { This work }\end{array}$ \\
\hline
\end{tabular}

of the wet crude oil emulsion directly influencing the bottle test repeatability [12]. To ensure the chemical integrity of the crude oil emulsion, no other chemicals, such as salts, solvents, acids or bases, were added to the sample.

- Ageing of the crude oil emulsion and demulsifier

Ageing of the crude oil emulsion significantly increases the viscosity due to a loss of the light hydrocarbons (e.-g., hexane, heptane, and pentane) and the partial oxidation of aromatics (e.g., phenol and cumene) and sulfur-containing molecules. This has a substantial effect on the bottle test repeatability [23]. All crude oil emulsion samples in this work were used within three days of sampling from the production facility. Demulsifier ageing was also shown to affect bottle test repeatability again due to the loss of light components in the demulsifier, such as methanol and light naphtha. Therefore, the demulsifiers in this study were used within one month of production.

- Mixing of crude oil emulsion and demulsifier

Different methods to mix the demulsifier with the crude oil emulsion were tested. The mixing required to incorporate the demulsifying agent cannot be too vigorous as the agitation may shear the emulsion and stabilize the emulsion. On the other hand, if the mixing is insufficient, little water separation will be obtained. The most reliable homogenization technique was by stirring the demulsifier in the crude oil emulsion using the displacement of the liquid by the vapor (mixture of air and light components from the emulsion) constituting the head space inside the vial. In other words, the sample bottles were rotated manually ( $2 \mathrm{~s}$ per cycle) for $10 \mathrm{~s}$. This step can also be performed using a vertical rotating mixer.

\section{- Temperature of bottle test}

The temperature source was kept low at $30{ }^{\circ} \mathrm{C}$ to reduce coalescence and accentuate the demulsifier effect on water separation. At higher temperatures, the slopes of the coalescence curves are greater leading to increase ambiguity in the interpretation of the bottle-test results for different conditions.

- Mass of demulsifier

The demulsifier dosage was measured by mass rather than by volume. The measurement of mass is more accurate than volume at the dosages required and is independent of temperature, density, vapor pressure, surface tension and viscosity of the demulsifier [26, 27].

- Repeatability of the bottle test

The repeatability of the bottle test was assessed by repeating the experiment two times. If the data points constituting each water separation curve was within the established level of confidence (97.5\%), the run was validated as acceptable. If not, the experiment was discarded and repeated. 
- Correction of the free water separated based on demulsifier dosage

When the precise demulsifier mass to achieve the target demulsifier dosage is not applied to the emulsion sample, and if the range of the resulting dosage is within +5 ppmw of the target dosage, the volume of free water separated $V_{e}^{S D}$ is corrected for the applied dosage. Within this small dosage range, the volume of water separated from the emulsion increases linearly with the demulsifier dosage. This correction accounts for the effect of demulsifier dosage variation on the demulsifier water separation performance. Hence, the volume of free water separated $V_{c}^{S D}$ from the bottle tests is corrected as:

$$
V_{c}^{S D}=\frac{m^{S D}}{m} \times V_{e}^{S D}
$$

where $m^{S D}$ and $m$ are the mass for the specified dosage and the mass added to the specific emulsion sample, respectively.

\subsection{Bottle test procedure}

Arab Medium crude oil emulsions were sampled from the production header of the production facility, upstream of chemical addition. The bottle tests were conducted within three days of sampling. The crude oil emulsion was re-homogenized by rotating the 1-L container five times, end-over-end for $3 \mathrm{~s}$ each cycle. The crude oil emulsion was added to six 100-mL glass tubes (See Fig. 1), at room temperature. The liquid level of emulsion in each tube was $100 \mathrm{~mL}$. Each demulsifier was properly mixed prior to dosing measurement. No solvent or diluent was added to the demulsifier. A 1-mL disposable plastic syringe was used to take approximately $0.2 \mathrm{~mL}$ of the demulsifier. The mass of the syringe with demulsifier was recorded. The demulsifier was dispensed to the $100-\mathrm{mL}$ tube containing the crude oil emulsion and the mass of the syringe was then measured using the balance. The difference in the syringe mass before and after dispensing the demulsifier gives an accurate measurement of the demulsifier mass $\left(m^{S D}\right)$ dispensed to the emulsion. A demulsifier concentration of $50 \mathrm{ppm}$ was added to five tubes. The sixth was used as reference. The mixing of the demulsifier with the emulsion was performed manually by rotating the tube, five times, endover-end for $2 \mathrm{~s}$ each cycle. Each tube was adjusted into a straight vertical position in a tube rack and placed in a thermostatic oven at $30^{\circ} \mathrm{C}$. The amount of free water separated was measured every $20 \mathrm{~min}$. The viscosity of these mixtures was not measured.

\subsection{Field-trial procedure for demulsifier selection}

A schematic of the wet crude oil handling section of a production facility is shown in Figure 2. Emulsion samples for the study were collected from crude oil sampling points upstream of the demulsifier injection. The sampling port is flushed for $10 \mathrm{~min}$ and a 1-L container is filled with the emulsion sample. The demulsifier is injected in the wet crude oil stream downstream of the production header at the entrance to the production facility. The wet crude oil stream enters a three-phase separator (Vessel 1) where gas, free water and crude oil are separated. The wet crude oil is heated in a heat exchanger and following a further decrease in pressure enters a second three-phase separator (Vessel 2). All separated gas and water are sent to gas and water gathering facilities.

The residence time of the crude oil stream through each section of the facility is noted in Figure 2. The residence time is important for consideration in the interpretation of bottle test results. The bottle test is a time-dependent batch experiment applied to the continuous process shown in Figure 2, where the residence time for the wet crude oil in the separation vessels is considered in the analysis of the test results. The main criterion for ranking demulsifiers in this work is the amount of water separated (water dropout) during the first $15 \mathrm{~min}$ of the bottle test experiment. This period of time corresponds approximately to the residence time of the wet crude oil from the demulsifier injection point to the outlet of Vessel 1. The period between 15 min and $60 \mathrm{~min}$ corresponds to the residence time from the outlet of Vessel 1 to the Vessel 2 outlet which is also of interest. The ranking of the demulsifier was not affected by the temperature difference between the bottle tests and the field trial. The field trials are sensitive to large variations in the process parameters. The field trials should be performed sequentially with little changes in temperature, wash water rate, interface and liquid levels to limit their impact on the dewatering. The diurnal temperature variation of the crude oil emulsion entering the Vessel 1 was $35 \pm 5^{\circ} \mathrm{C}$. The crude oil emulsion is heated to $46 \pm 2{ }^{\circ} \mathrm{C}$ before entering the Vessel 2 (Fig. 2) and water separation mostly occurred in the Vessel 2, the effect of process temperature on the demulsifiers was minimal during the field trials. Flowmeters are key instruments during demulsifier field trial to measure and monitor the addition of demulsifier $\left(F_{d}\right.$ in Fig. 2$)$ and the water separated from the separator vessels $\left(F_{1}, F_{2}\right.$ and $F_{D}$ in Fig. 2). Flow rate measurements in the field are by orifice differential pressure flowmeters. Flowmeter reliability is a key variable in the field test and the orifice plate flow meter is robust, has good accuracy and is easy to maintain. The demulsifiers tested were injected upstream of Vessel 1. Additionally, the incumbent demulsifier was injected at the dehydrator inlet to minimize production upsets caused by poor separation. The fractional water separation from the three multiphase separators (see Fig. 2) is a primary quantification of the water separation performance or demulsifier efficiency. Although the production facility process and instruments vary, the field trial procedure remains the same.

The initial demulsifier dosage is 5 vol\% greater than the incumbent dosage. The first dosage of the tested demulsifier is maintained for at least one day to ensure that no traces of the previous demulsifier remain in the process. The demulsifier dosage is subsequently decreased in steps of $10 \mathrm{ppm}$ starting from the initial dosage of the course of several days. This procedure continues until a dehydrator upset occurs. 


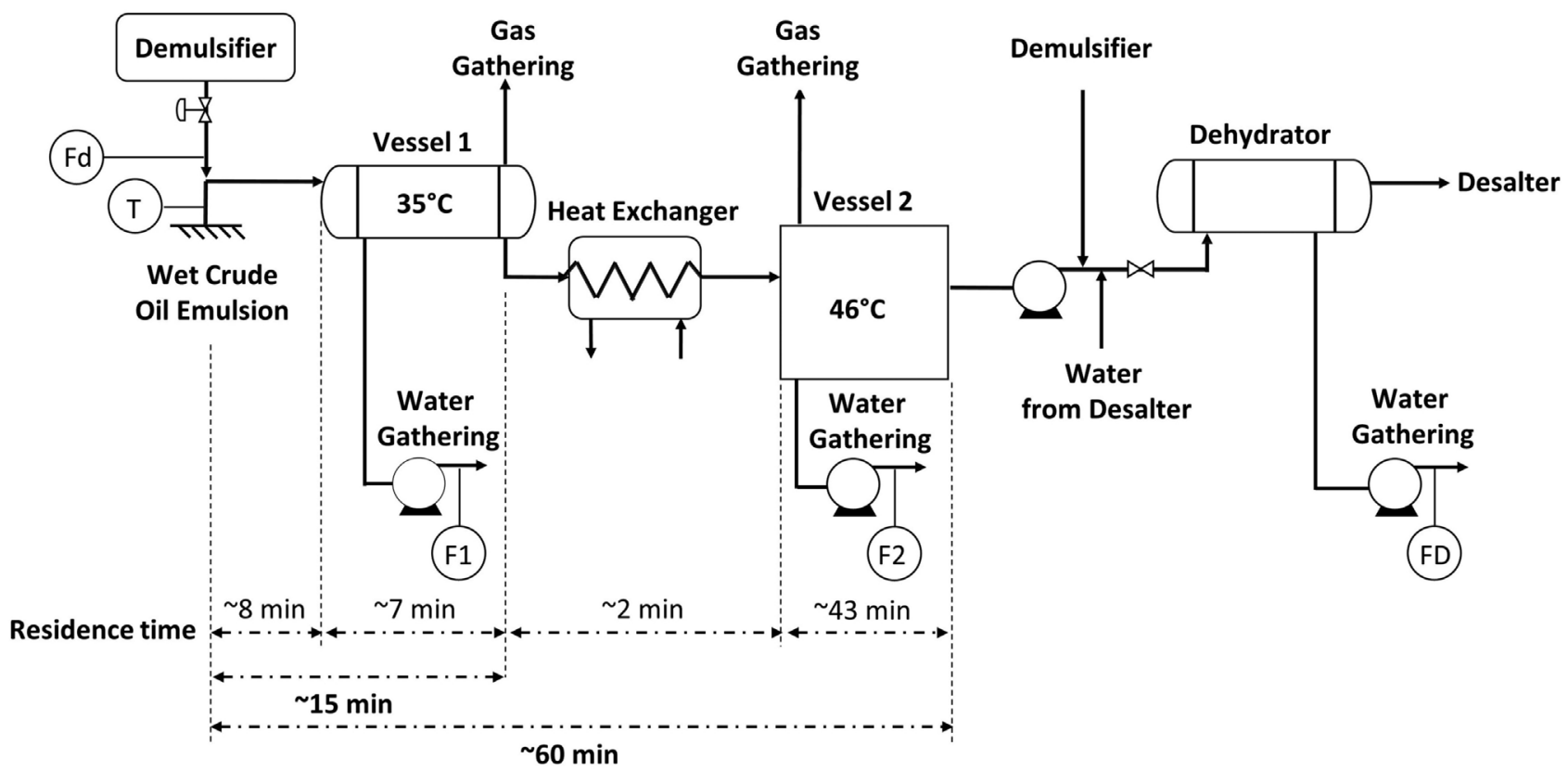

Fig. 2. Schematic of the wet crude oil handling section of the production facility.

The dehydrator upset occurs when there is rapid change in the dehydrator grid voltage caused by an increase in conductivity due to poor water separation. The demulsifier dosage is increased slightly above the dosage that caused dehydrator upset. At the end of the field trial, the water separation efficiency, the percentage of the total water separated from Vessel 1, $F_{1} /\left(F_{1}+F_{2}+F_{D}\right)$, and Vessel 2, $F_{2} /\left(F_{1}+F_{2}+F_{D}\right)$, is calculated as a function of demulsifier dosage.

\section{Results and discussion}

\subsection{Bottle test results}

Four demulsifiers were tested for their water separation performance at $30{ }^{\circ} \mathrm{C}$ using the methodology for bottle-test experiments described in the previous section. Figure 3 shows the water separation performance as the percentage of water separated with time of the four demulsifiers at a dosage of 50 ppmw. The error bars on Figure 3 represent the range of interface level measurements in each bottle-test experiment and on average was $\pm 1.25 \%$. Demulsifiers A and $\mathrm{C}$ show the highest water separation after $60 \mathrm{~min}$ and were selected for field-testing along with the incumbent demulsifier (Demulsifier B). The water quality from all four demulsifiers was qualitatively excellent with no oil droplets on the bottle/tube wall and clear water with no coloration.

\subsection{Field-trial optimization of demulsifier dosage}

The demulsifier dosage was varied during the field trial to observe the demulsifier dosage effect on the water separation.

\subsubsection{Demulsifier B (incumbent demulsifier) field trial}

The Demulsifier B dosage (incumbent demulsifier) and the percentage of water draw-off from Vessel 2 and the Dehydrator during the field trial are plotted in Figure 4. No significant water separation was observed in Vessel 1 during the field trial. The water draw-off is calculated as:

$\%$ Water draw - off $=$

$$
\frac{\text { Flowrate of water separated from a vessel }}{\text { Total inlet flowrate of wet crude oil emulsion }} \text {. }
$$

Figure 4 shows that the time-varying profile of the Vessel 2 draw-off remains relatively constant as the demulsifier dosage is decreased in decrements of $10 \mathrm{ppm}$. A large drop in draw-off occurs from the $30 \mathrm{ppm}$ to $25 \mathrm{ppm}$ target dosage. The target dosage is the dosage inputted by the operator. The water draw-off from the downstream dehydrator vessel increases as a consequence. Table 3 lists the target and actual demulsifier dosage and the average water draw-off from Vessel 2 during the field trial of Demulsifier B.

Figure 4 and Table 3 also show that the demulsifier target dosage can differ from the actual dosage during operation because of the process dynamics. The actual dosage is the dosage recorded by a calibrated flowmeter.

There is little change in the average water draw-off from Vessel 2 when decreasing the demulsifier dosage from $50 \mathrm{ppm}$ to $30 \mathrm{ppm}$. It is likely that there is a residual accumulation of the demulsifier in the process following the relatively high $50 \mathrm{ppm}$ dosage. The field trial protocol improved for subsequent field trials by changing the dosing procedure from decreasing the dosage to increasing the dosage so that the effect of residual demulsifier accumulation 


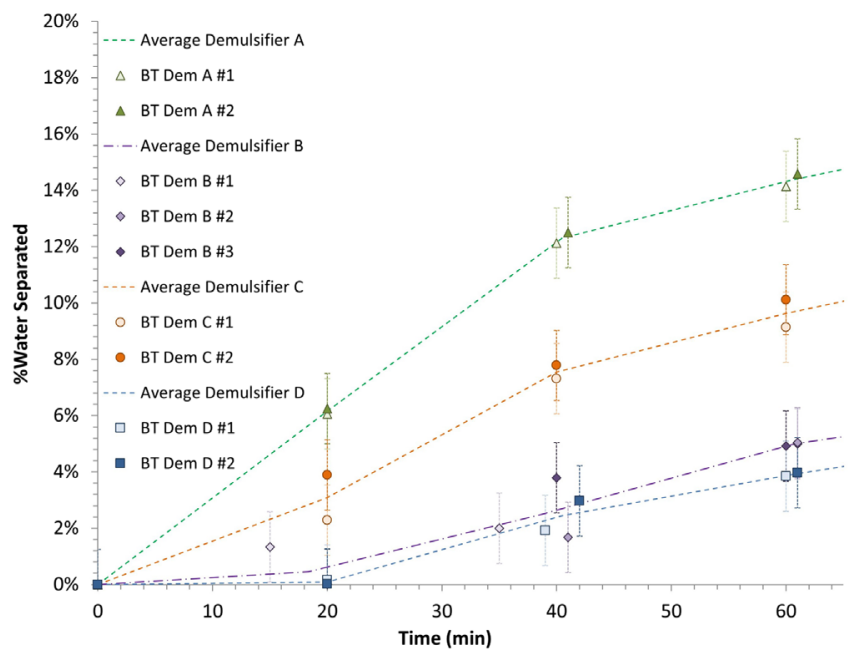

Fig. 3. Water separation performance of four demulsifiers at $30{ }^{\circ} \mathrm{C}$ (dosage of $50 \mathrm{ppmw}$ ).

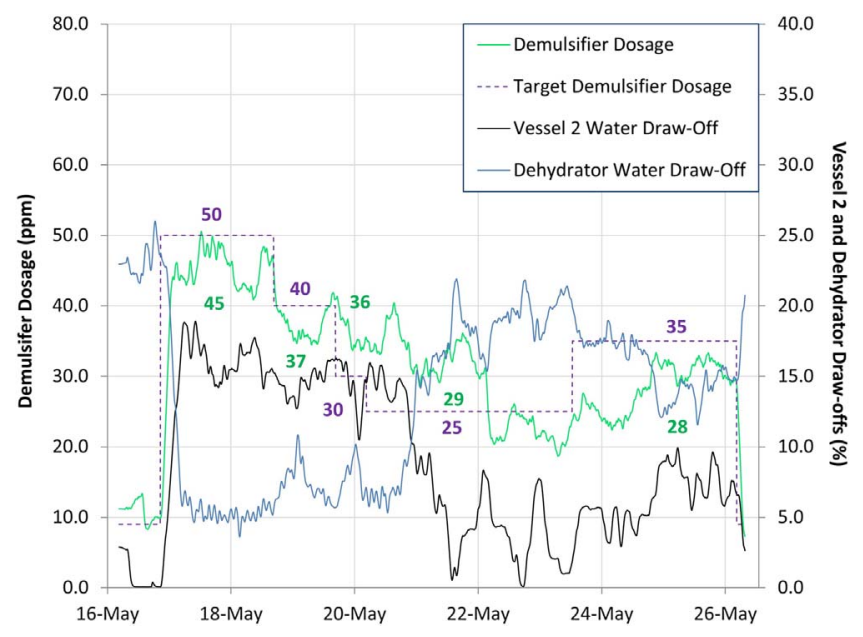

Fig. 4. Demulsifier target dosage and percentage of water drawoff from Vessel 2 and the dehydrator during the field trial of Demulsifier B.

is eliminated. In Table 4, the average water draw-off from Vessel 2 is shown whereby the water draw-off is averaged during a period of relatively constant or steady water draw-off from the dehydrator. The average water draw-off reached $14 \%$ in Vessel 2 for an average demulsifier concentration of $36 \mathrm{ppm}$. For a dosage of $45 \mathrm{ppm}$, little improvement in water separation is observed. The Demulsifier B bottle test showed approximately $5 \mathrm{vol} \%$ water separated with $50 \mathrm{ppm}$ of demulsifier after $60 \mathrm{~min}$. This result is compared with the average water draw-off of $16 \mathrm{vol} \%$ from Vessel 2 at a 45 ppm dosage of Demulsifier B. The bottle-test water separation performance of Demulsifier B is significantly lower than the field-trial water separation performance. It is well known that vessel hydrodynamics and the presence of light components generally lead to a lower demulsifier dosage in field trials as compared to bottle tests. However, the discrepancy between the field-trial and
Table 3. Average water draw-off for different dosages of Demulsifier B.

\begin{tabular}{lccc}
\hline Time & $\begin{array}{c}\text { Target } \\
\text { demulsifier } \\
\text { dosage } \\
\text { (ppm) }\end{array}$ & $\begin{array}{c}\text { Actual } \\
\text { demulsifier } \\
\text { dosage } \\
\text { (ppm) }\end{array}$ & $\begin{array}{c}\text { Average } \\
\text { water } \\
\text { draw-off } \\
\text { from } \\
\text { Vessel 2 (\%) }\end{array}$ \\
\hline 17-May 3:30 & 9 & $11 \pm 2$ & 0 \\
17-May 16:00 & 50 & $45 \pm 4$ & 15 \\
19-May & 40 & $37 \pm 3$ & 15 \\
20-May & 30 & $36 \pm 2$ & 14 \\
21-May & 25 & $29 \pm 6$ & 6 \\
24-May & 35 & $28 \pm 4$ & 6 \\
\hline
\end{tabular}

bottle-test results is also likely due to a change in composition with time of Demulsifier B caused by evaporation of the demulsifier solvents. Figure 5 shows the lack of homogeneity in the Demulsifier B sample with a layer of a white solid at the container bottom and a dark brown layer on the top. Demulsifiers A and C were a homogenous dark orange solution. Bottle tests performed with Demulsifier B extracted from below the surface, and from the middle of the sample bottle gave different amounts of water separation.

\subsubsection{Demulsifier C field trial}

A new procedure was developed to prevent the accumulation of residual demulsifier following a step-change in dosage. The initial demulsifier dosage is $20 \mathrm{ppm}$. The dosage is incremented by $10 \mathrm{ppm}$ daily. After the original incumbent dosage plus $5 \%$ is reached, the dosage is reduced stepwise to $30 \mathrm{ppm}$ and then to $20 \mathrm{ppm}$. The Demulsifier C field trial was performed using this improved field-trial procedure.

Figure 6 shows the water draw-off and demulsifier dosage during the field trial. The average quantities are summarized in Table 5. At a low dosage of 17-26 ppm, there is approximately $1 \%$ water separation from the Vessel 2. When the demulsifier dosage is increased to $34 \mathrm{ppm}$, the water draw-off increases to $5 \%$.

Referring to Table 5 , a dosage of $42 \mathrm{ppm}$ and $58 \mathrm{ppm}$, produced water draw-off of $9 \%$ and $11 \%$, respectively. No significant water separation was observed in Vessel 1 during the entire period of this field trial. When the demulsifier dosage was decreased from $58 \mathrm{ppm}$ to $26 \mathrm{ppm}$, a significant accumulation effect is observed on the water drop-off, as shown by the yellow box in Figure 6 . The water draw-off slowly decreases following the step change in demulsifier rate illustrating the demulsifier accumulation effect. There is a substantial residual concentration of demulsifier present in the process. In the separator vessels, the residence time is distributed over a wide range. The circulation patterns in Vessel 1 are known to promote short circuiting from the inlet to the outlet but also present a long tail in the residence time distribution. This distribution is likely the reason for the under-damped response in the Vessel 2 water 
Table 4. Average water draw-off for different dosages of Demulsifier B.

\begin{tabular}{lcc}
\hline Time & $\begin{array}{c}\text { Actual demulsifier } \\
\text { dosage }(p p m)\end{array}$ & $\begin{array}{c}\text { Average water draw-off } \\
\text { from Vessel 2 }(\%)\end{array}$ \\
\hline 17-May 03:30 - 17-May 14:10 & $11 \pm 2$ & 0 \\
18-May 00:00 - 19-May 11:10 & $45 \pm 4$ & 16 \\
19-May 23:50 - 20-May 02:20 & $37 \pm 3$ & 15 \\
20-May 21:50 - 21-May 10:40 & $36 \pm 2$ & 15 \\
24-May 13:00 - 25-May 12:20 & $25 \pm 2$ & 5 \\
\hline
\end{tabular}

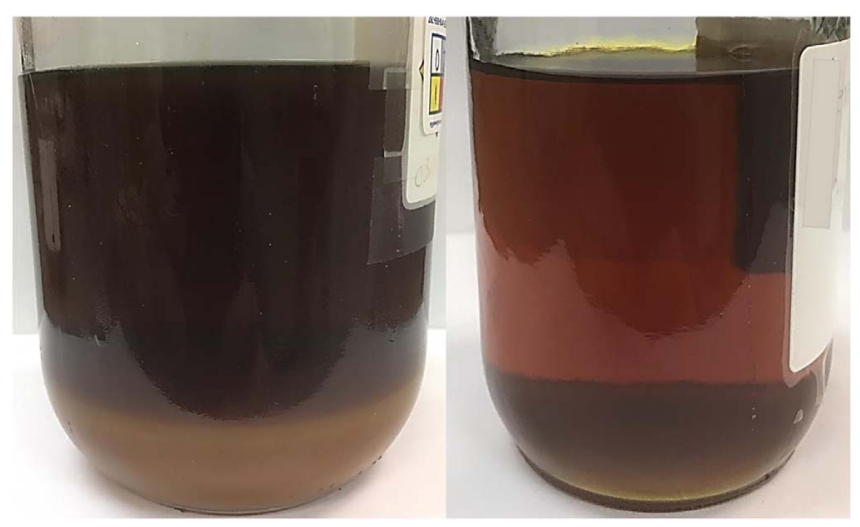

Fig. 5. Picture of Demulsifier B showing a white precipitate (right) and Demulsifier A showing a clear solution (left).

draw-off. Holding the demulsifier dosage constant for a period of 2 or more days would see the water draw-off stabilize. This is not desirable as the production facility would prefer to compress the demulsifier testing into the shortest period allowable. The average of water drained from Vessel 2 was adjusted to correct the accumulation effect of the system by identifying area of process stability. The accumulation effect wasn't observed, when the demulsifier dosage was increased. The average of water drained from the Vessel 2 is $1.5 \%$ for an average of $26 \mathrm{ppm}$ (4-May in Tab. 5) and $1 \%$ for an average of $18 \mathrm{ppm}$ (5-May in Tab. 5), which correlates well with value at the beginning of the field trial: $1 \%$ for an average of $26 \mathrm{ppm}$ (29-Apr in Tab. 5) and $1 \%$ for an average of $17 \mathrm{ppm}$ (27-Apr in Tab. 5).

The Demulsifier C bottle test results (see Fig. 3) are in good agreement with the field trial. In the bottle test, approximately $10 \mathrm{vol} \%$ water was separated with $50 \mathrm{ppm}$ of demulsifier after $60 \mathrm{~min}$. In the field trial, the water draw-off from Vessel 2 was 9-11 vol\% for 42 and 58 ppm dosage of Demulsifier C, respectively.

Future demulsifier field trials must be conducted with demulsifier dosage increments rather than dosage decrements to prevent false results from the delayed change in demulsifier concentration due to accumulation in the process.

\subsubsection{Demulsifier A field trial}

The Demulsifier A field trial was performed using the improved field-trial procedure applied in the Demulsifier C

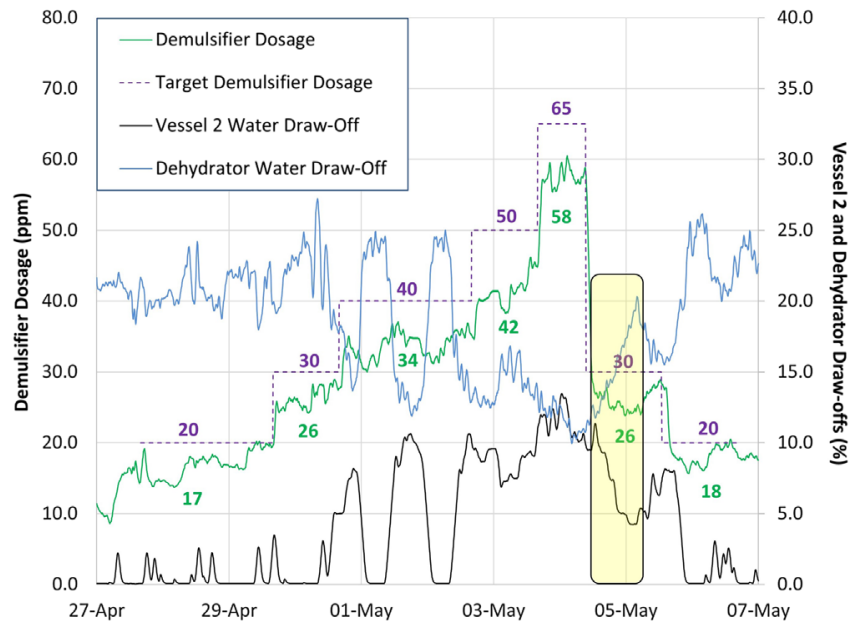

Fig. 6. Demulsifier dosage and percentage of water draw-off from Vessel 2 and the Dehydrator during the field trial of Demulsifier C. The yellow region identifies the demulsifier accumulation effect.

field trial and described in the previous section. Figure 7 shows the water draw-off and demulsifier dosage during the field trial. The average quantities are summarized in Table 6 .

In Table 6 , there is approximately $2 \%$ water separation observed in Vessel 2 at a low demulsifier dosage of $18 \mathrm{ppm}$. When the demulsifier dosage is increased to $29-32 \mathrm{ppm}$, the water separation increases significantly to approximately $10-11 \%$. A small water separation of $8 \%$ water draw-off was observed in Vessel 1 at a demulsifier dosage of $45 \mathrm{ppm}$. The total water draw-off from Vessel 1 and Vessel 2 increased to $13 \%$. When the demulsifier dosage is increased to $63 \mathrm{ppm}$, the water separation performance increased even further to $16 \%$ total water separated from Vessel 1 and Vessel 2, significantly decreasing the load on the dehydrator. The accumulation effect wasn't observed, when the demulsifier dosage was increased. When the dosage was reduced from $63 \mathrm{ppm}$ to $29 \mathrm{ppm}$ and then to $22 \mathrm{ppm}$, the water draw-off remained at a stable level of $12 \%$ from the Vessel 2. The demulsifier accumulation effect in the process was greatest with Demulsifier A, compared to demulsifiers B and $\mathrm{C}$, because the process didn't stabilize even after 1 day. For the last two demulsifier dosages of this field trial (28-Aug and 29-Aug in Tab. 6), a correction for the accumulation effect was not possible as the accumulation effect 
Table 5. Average water draw-off for different dosages of Demulsifier C.

\begin{tabular}{lccc}
\hline Time & $\begin{array}{c}\text { Target demulsifier } \\
\text { dosage }(\mathrm{ppm})\end{array}$ & $\begin{array}{c}\text { Actual demulsifier } \\
\text { dosage }(\mathrm{ppm})\end{array}$ & $\begin{array}{c}\text { Average water draw-off } \\
\text { from Vessel 2 }(\%)\end{array}$ \\
\hline 27-Apr & 20 & $17 \pm 3$ & 1 \\
29-Apr & 30 & $26 \pm 2$ & 1 \\
30-Apr & 40 & $34 \pm 3$ & 5 \\
2-May & 50 & $42 \pm 4$ & 9 \\
3-May & 65 & $58 \pm 4$ & 11 \\
4-May & 30 & $26 \pm 2$ & $7(\text { corr. } 1.5 \%)^{\mathrm{a}}$ \\
5-May & 20 & $18 \pm 3$ & $3(\text { corr. } 1 \%)^{\mathrm{a}}$ \\
\hline
\end{tabular}

${ }^{a}$ Average of water drained from Vessel 2 when the accumulation of demulsifier in the process is taken into consideration.

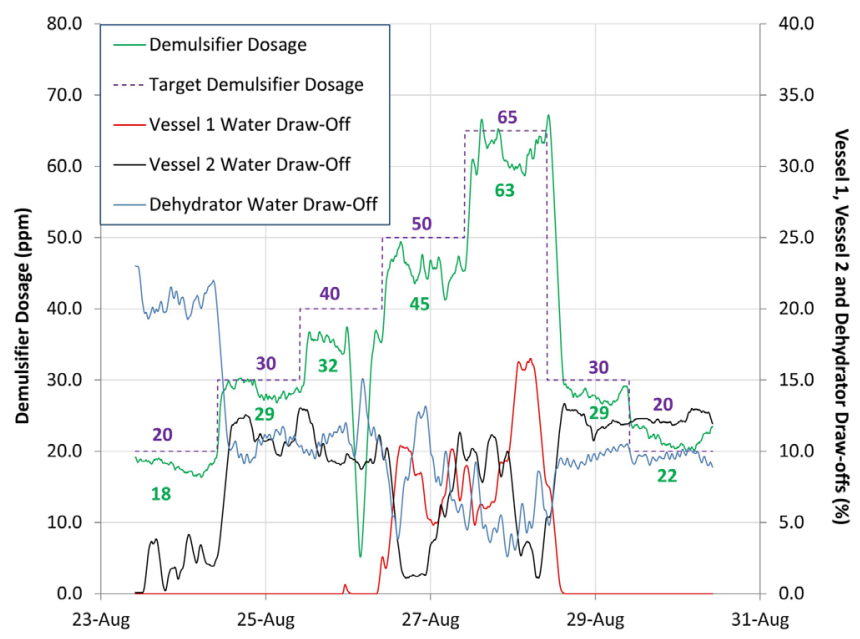

Fig. 7. Demulsifier dosage and percentage of water draw-off from Vessel 1, Vessel 2 and the dehydrator during the field trial of Demulsifier A.

was present for the full day between set point changes in the dosage.

Similarly to Demulsifier $\mathrm{C}$, the bottle test results for Demulsifier A were in good agreement with the field trial. The bottle test gave approximately $14 \mathrm{vol} \%$ of water separation at a $50 \mathrm{ppm}$ of demulsifier dosage after $60 \mathrm{~min}$, while the field trial had a total water draw-off for Vessels 1 and 2 of 13-16 vol\% for 45 and $63 \mathrm{ppm}$ dosage of Demulsifier A, respectively. The accumulation effect is shown to have a significant influence on water separation when the demulsifier dosage is decreased during the field trials.

\subsection{Mathematical interpretation of the demulsifier performance}

Figure 8 plots the average water draw-off during the trial as a function of the demulsifier dosage and illustrates the demulsifier water separation performance. The demulsifier performance of increased water draw-off with increased demulsifier dosage can be curve-fit with a sigmoidal function. Demulsifier A was the only demulsifier that produced water separation in Vessel 1, while demulsifiers B and C caused water separation only in Vessel 2. Figure 9 also shows the water drained from both vessels and the total, using Demulsifier A.

A sigmoid or "S-curve" is commonly used to describe kinetic curves. The results in Figures 8 and 9 show that a sigmoid curve is the best suited mathematical description for the effect of demulsifier dosage. The sigmoid is described in three phases: a lag phase, a growth phase and a final equilibrium phase or plateau $[28,29]$. The lag phase is a period of low water separation caused by a concentration of demulsifier below the minimum dosage necessary to have an effective action. The demulsifier concentration is too low to cause a significant change in water draw-off. The quantity of demulsifier molecules on water droplets dispersed in the crude oil emulsion are insufficient to cause significant coalescence. The growth phase is the phase in which the demulsifier effectiveness increases. After the inflexion point, the rate of change of water draw-off decelerates (second derivative) leading to the equilibrium plateau. The demulsifier action is limited by an excessive concentration of demulsifier molecules (saturation). It has been reported that a decrease in separation performance can occur as the demulsifier concentration increases due to the reverse demulsifier action of stabilizing the emulsion droplets $[4,30-32]$. This effect is not seen in Figure 8, as the water draw-off plateaus to the demulsifier dosages employed in the field trials.

A mathematical analysis of the third derivative, or jerk, of the sigmoid curve indicates that there is one minimum at the inflexion point and two maxima, one in the lag phase and one at the end of the growth phase. The maxima of the jerk are points of interest to the optimization of demulsifier dosage. The first maximum corresponds to the point in which the minimum effective demulsifier dosage is necessary to see $1 \%$ of water separation. The demulsifier dosage at this point can be graphically determined by the intersection of the tangent to the growth slope and the value of zero water separation ( $x$-axis). The second maximum of the jerk defines the limit situations in which any further increase in concentration will not lead to an increase in water separation. The demulsifier concentration at this limiting value can be graphically determined as the intersection of the tangent to the plateau (maximum separation) and the tangent to the slope at the inflection point (maximum 
Table 6. Average water draw-off for different dosages of Demulsifier A.

\begin{tabular}{lccccc}
\hline Time started & $\begin{array}{c}\text { Target } \\
\text { demulsifier } \\
\text { dosage (ppm) }\end{array}$ & $\begin{array}{c}\text { Actual } \\
\text { demulsifier } \\
\text { dosage (ppm) }\end{array}$ & $\begin{array}{c}\text { Average water } \\
\text { draw-off from } \\
\text { Vessel 1 (\%) }\end{array}$ & $\begin{array}{c}\text { Average water } \\
\text { draw-off from } \\
\text { Vessel 2 (\%) }\end{array}$ & $\begin{array}{c}\text { Total water } \\
\text { draw-off from } \\
\text { Vessel 1 \& 2 (\%) }\end{array}$ \\
\hline 23 -Aug & 20 & $18 \pm 2$ & 0 & 2 & 2 \\
24 -Aug & 30 & $29 \pm 2$ & 0 & 11 & 11 \\
25 -Aug & 40 & $32 \pm 8$ & 0 & 10 & 10 \\
26 -Aug & 50 & $45 \pm 4$ & 8 & 5 & 13 \\
27 -Aug & 65 & $63 \pm 5$ & 9 & 12 & 12 \\
28-Aug & 30 & $29 \pm 5$ & 0 & 12 & 12 \\
29-Aug & 20 & $22 \pm 2$ & 0 & & 12 \\
\hline
\end{tabular}

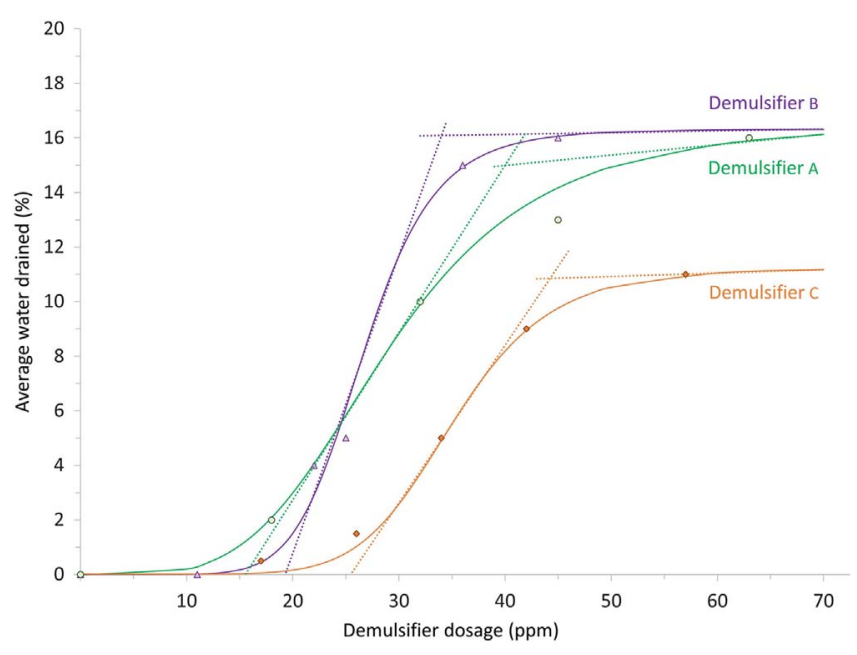

Fig. 8. Average water draw-off as a function of demulsifier dosage.

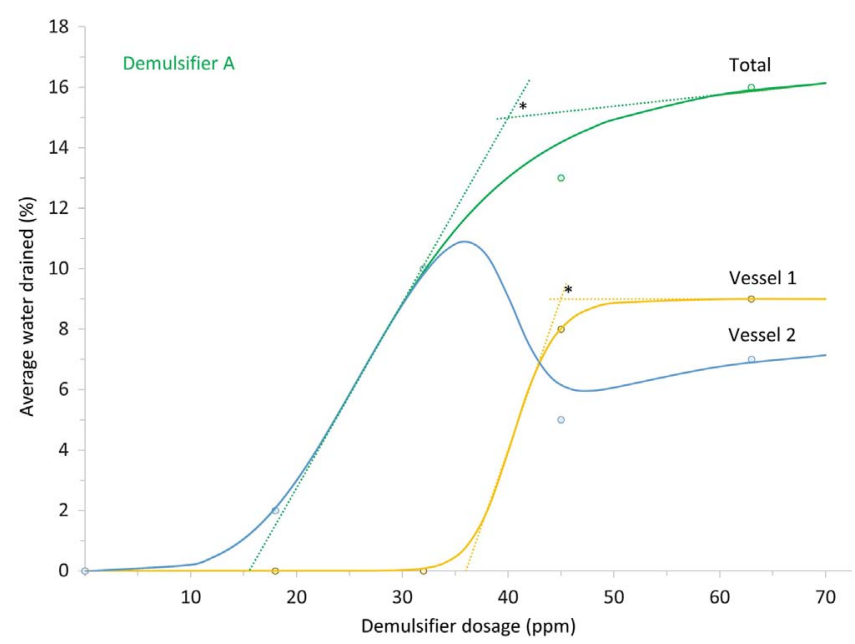

Fig. 9. Combination of water draw-off in Vessel 1 and Vessel 2 for Demulsifier A.

efficiency or speed). A simple sigmoidal fit can be employed for demulsifiers A, B and C on Figure 8, as well as for the Vessel 1 for Demulsifier A. This is not the case for Vessel 2 with Demulsifier A, as shown on Figure 9.
Table 7. Minimum and maximum demulsifier dosage of different demulsifiers tested in this study.

\begin{tabular}{lcc}
\hline Demulsifier & $\begin{array}{c}\text { Minimum } \\
\text { dosage (ppm) }\end{array}$ & $\begin{array}{c}\text { Maximum } \\
\text { dosage (ppm) }\end{array}$ \\
\hline Demulsifier A & 15 & 40 \\
Demulsifier A (Vessel 1) & 36 & 45 \\
Demulsifier B & 19 & 34 \\
Demulsifier C & 25 & 44 \\
\hline
\end{tabular}

With the goal of optimizing demulsifier dosage to maximize water separation, the maximum demulsifier dosage is determined graphically from Figure 8. For a demulsifier dosage set point below the minimum demulsifier dosage, little or no water separation will occur wasting the demulsifier.

The minimum, maximum and most efficient values for the tested demulsifiers are summarized in Table 7. This information may be used to determine the optimum demulsifier dosage required to achieve a specific water concentration at the dehydrator inlet which has a positive economic benefit to the facility operations.

\section{Conclusion}

This study develops a strategy to improve the bottle test and the reliability of the demulsifier evaluation and selection for dewatering wet crude oil emulsions. The following conclusions were obtained:

- Good agreement between the bottle tests and the field trials was obtained by improving the commonly practiced methods with the exception of the inhomogeneous demulsifier.

- With the following improvements to the bottle test experimental protocol, a confidence level of $97.5 \%$ is achieved in repeatability:

- The chemical integrity of the emulsion and demulsifier is maintained by not using diluents or adding any other chemicals.

- The ageing of samples is minimized with three days for the emulsion and one month for the demulsifier. 
- The bottle-test temperature is optimized for maximum water separation effect.

- Demulsifier dosage errors are significantly minimized by measuring the demulsifier mass and by correcting the separated water volume by multiplying with the ratio of specified dosage mass and the actual mass added to the emulsion sample.

- During the demulsifier field trials the dosage is increased and not decreased to avoid the demulsifier accumulation effect when changing from higher to lower dosage as shown from the data.

- A mathematical model was used to describe the demulsifier water separation performance. A simple method to determine the optimal demulsifier range was established using graphical analysis.

- The application of the improved field-trial methodology may be used to determine the optimal demulsifier dosage to achieve a desired water concentration in the wet crude oil to the dehydrator inlet.

Acknowledgments. This work is the result of a collaboration between the Saudi Aramco Research \& Development Center and the Northern Area Oil Operations. The authors would like to acknowledge Dr. Regis Vilagines and Dr. Simone Less for their support; and would like to especially thank, Ellyes Mecikar, for his support and his valuable contributions to the progress of this work. The authors would also like to thank Mr. Philip Embleton for his support in reviewing the manuscript for submission.

\section{References}

1 Sjöblom J., Aske N., Auflem I.H., Brandal Ø., Havre T.E., Sæther Ø., Westvik A., Johnsen E.E., Kallevik H. (2003) Our current understanding of water-in-crude oil emulsions: Recent characterization techniques and high pressure performance, Adv. Colloid Interface Sci. 100, 399-473. https://doi.org/10.1016/S0001-8686(02)00066-0.

2 Harr J.D. (1969) Field approach to crude oil demulsification, SPE, 1-9. https://www.onepetro.org/general/SPE-2694-MS.

3 Thompson D.G., Taylor A.S., Graham D.E. (1985) Emulsification and demulsification related to crude oil production, Colloids Surf. 15, 175-189. https://doi.org/10.1016/01666622(85)80070-6.

4 Zolfaghari R., Fakhru'l-Razi A., Abdullah L.C., Elnashaie S. S.E.H., Pendashteh A. (2016) Demulsification techniques of water-in-oil and oil-in-water emulsions in petroleum industry, Sep. Sci. Technol. 170, 377-407. https://doi.org/ 10.1016/j.seppur.2016.06.026.

5 Kilpatrick P.K. (2012) Water-in-crude oil emulsion stabilization: Review and unanswered questions, Energy Fuels 26, 7, 4017-4026. https://doi.org/10.1021/ef3003262.

6 Kumar K., Nikolov A.D., Wasan D.T. (2001) Mechanisms of stabilization of water-in-crude oil emulsions, Ind. Eng. Chem. Res. 40, 14, 3009-3014. https://doi.org/10.1021/ie000663p.

7 Bowman R.W., Burton W.D., Pryor J.A. (1977) Statistically designed oil dehydration tests, in: California Regional Meeting, Bakersfield, California.

8 Mohammed R.A., Bailey A.I., Luckham P.F., Taylor S.E. (1993) Dewatering of crude oil emulsions 2. Interfacial properties of the asphaltic constituents of crude oil, Colloids Surf. A Physicochem. Eng. Asp. 80, 2-3, 237-242. https://doi.org/10.1016/0927-7757(93)80203-Q.

9 Jones T.J., Neustadter E.L., Whitting K.P. (1978) Water-incrude oil emulsion stability and emulsion destabilization by chemical demulsifiers, J. Can. Pet. Technol. 17, 2, 100-108. https://doi.org/10.2118/78-02-08.

10 Less S., Vilagines R. (2012) The electrocoalescers' technology: Advances, strengths and limitations for crude oil separation, J. Pet. Sci. Eng. 81, 57-63. https://doi.org/ 10.1016/j.petrol.2011.12.003.

11 McLean J.D., Kilpatrick P.K. (1997) Effects of asphaltene solvency on stability of water-in-crude-oil emulsions, J. Colloid Interface Sci. 189, 2, 242-253. https://doi.org/ 10.1006/jcis.1997.4807.

12 Manning F.S., Thompson R.E. (1995) Dehydration of crude oil, in: Oilfield processing volume two: Crude oil, Penn Well Corporation, Tulsa, Oklahoma, pp. 113-143. ISBN 13: 9780878143542.

13 Kokal S.L. (2005) Crude oil emulsions: A state-of-the-art review, SPE Prod. Facil. 20, 1, 5-13. https://doi.org/ 10.2118/77497-PA.

14 Graham D.E., Stockwell A. (1980) Selection of demulsifiers for produced crude oil emulsions, SPE, 453-458. https://doi. org/10.2118/191-1980-MS.

15 Goldszal A., Bourrel M. (2000) Demulsification of crude oil emulsions: Correlation to microemulsion phase behavior, Ind. Eng. Chem. Res. 39, 8, 2746-2751. https://doi.org/10.1021/ ie990922e.

16 ASTM Standard D4052-18a (2019) Standard test method for density, relative density, and API gravity of liquids by digital density meter, ASTM International, West Conshohocken, PA, USA. https://doi.org/10.1520/D4052-18A.

17 Zargar G., Gheysari R.G., Takassi M.A., Rostami A., Zadehnazari A. (2018) Evaluation of a sulfanilic acid based surfactant in crude oil demulsification: an experimental study, Oil Gas Sci. Technol. - Rev IFP Energies nouvelles 73, 20. https://doi.org/10.2516/ogst/2018016.

18 Wang X., Alvarado V. (2012) Effects of aqueous-phase salinity on water-in-crude oil emulsion stability, J. Dispers. Sci. Technol. 33, 165-170. https://doi.org/10.1080/ 01932691.2010 .548689 .

19 Borges B., Rondon M., Sereno O., Asuaje J. (2009) Breaking of water-in-crude-oil emulsions. 3. Influence of salinity and water-oil ratio on demulsifier action, Energy Fuels 23, 1568 1574. https://doi.org/10.1021/ef8008822.

20 Silva I., Borges B., Blanco R., Rondon M., Salager J.-L., Pereira J.C. (2014) Breaking of water-in-crude oil emulsions. 5. Effect of acid-alkaline additives on the performance of chemical demulsifiers, Energy Fuels 28, 6, 3587-3593. https://doi.org/10.1021/ef5006918.

21 Hajivand P., Vaziri A. (2015) Optimization of demulsifier formulation for separation of water from crude oil emulsions, Braz. J. Chem. Eng. 32, 1, 107-118. https://doi.org/ 10.1590/0104-6632.20150321s00002755.

22 Acrivos A., Herbolzheimer E. (1979) Enhanced sedimentation in settling tanks with inclined walls, J. Fluid Mech. 92, 3, 435-457. https://doi.org/10.1017/S0022112079000720.

23 Filho D.C.M., Ramalho J.B.V.S., Spinelli L.S., Lucas E.F. (2012) Aging of water-in-crude oil emulsions: Effect on water content, droplet size distribution, dynamic viscosity and stability, Colloids Surf. A Physicochem. Eng. Asp. 396, 208-212. https://doi.org/10.1016/j.colsurfa.2011.12.076. 
24 Salam K.K., Alade A.O., Arinkoola A.O., Opawale A. (2013) Improving the demulsification process of heavy crude oil emulsion through blending with diluent, J. Pet. Eng. 2013, 1-6. https://doi.org/10.1155/2013/793101.

25 Rondon M., Pereira J.C., Bouriat P., Graciaa A., Lachaise J., Salager J.-L. (2008) Breaking of water-in-crude-oil emulsions. 2. Influence of asphaltene concentration and diluent nature on demulsifier action, Energy Fuels 22, 702-707. https://doi.org/10.1021/ef7003877.

26 Feldmann R., Lochner K.H. (2015) Influences on volume in piston-operated air-displacement pipettes, Accred. Qual. Assur. 21, 1, 69-82. https://doi.org/10.1007/s00769-015-1171-y.

27 Lochner K.H., Ballweg T., Fahrenkrog H.-H. (1996) Untersuchungen zur Meßgenauigkeit von Kolbenhubpipetten mit Luftpolster [Factors influencing the measuring accuracy of piston pipettes with air interface], J. Lab. Med. 20, 7/8, 430-440. https://doi.org/10.1515/labm.1996.20.7-8.430.

28 Bentea L., Watzky M.A., Finke R.G. (2017) Sigmoidal nucleation and growth curves across nature fit by the Finke-Watzky model of slow continuous nucleation and autocatalytic growth: Explicit formulas for the lag and growth times plus other key insights, J. Phys. Chem. C 121, 9, 5302-5312. https://doi.org/10.1021/acs.jpcc.6b12021.

29 Uversky V.N., Li J., Fink A.L. (2001) Evidence for a partially folded intermediate in $\alpha$-synuclein fibril formation, J. Biol. Chem. 276, 14, 10737-10744. https://doi.org/ 10.1074/jbc.M010907200.

30 Nguyen D., Sadeghi N., Houston C. (2012) Chemical interactions and demulsifier characteristics for enhanced oil recovery applications, Energy Fuels 26, 5, 2742-2750. https://doi.org/10.1021/ef201800b.

31 Al-Sabagh A.M., Kandile N.G., Noor El-Din M.R. (2011) Functions of demulsifiers in the petroleum industry, Sep. Sci. Technol. 46, 7, 1144-1163. https://doi.org/10.1080/01496395. 2010.550595.

32 Mohammed R.A., Bailey A.I., Luckham P.F., Taylor S.E. (1993) Dewatering of crude oil emulsions 1. Rheological behaviour of the crude oil-water interface, Colloids Surf. A Physicochem. Eng. Asp. 80, 2-3, 223-235. https://doi.org/ 10.1016/0927-7757(93)80202-P. 